\title{
Photoemission Spectra of Sound Tooth and Those of Different Carious Stages
}

\author{
Ali Abdel-Rahman Saeed Marouf ${ }^{1, *}$, Yathrib Awad Khairallah ${ }^{2}$, Elhadi Mohieldin Awooda ${ }^{3}$ \\ ${ }^{1}$ Institute of Laser, Sudan University of Science and Technology, Khartoum, Sudan \\ ${ }^{2}$ College of Science, Sudan University of Science and Technology, Khartoum, Sudan \\ ${ }^{3}$ Department of Restorative Dentistry, Faculty of Dentistry, University of Medical Sciences and Technology (UMST), Khartoum, Sudan
}

Email address:

marouf.44@gmail.com (A. Abdel-Rahman S. M.), alisaeed@sustech.edu (A. Abdel-Rahman S. M),

yathrib617@gmail.com (Y.A. Khairallah),sowaraldahb@gmail.com (E. M. Awooda)

${ }^{*}$ Corresponding author

\section{To cite this article:}

Ali Abdel-Rahman Saeed Marouf, Yathrib Awad Khairallah, Elhadi Mohieldin Awooda. Photoemission Spectra of Sound Tooth and Those of Different Carious Stages. European Journal of Biophysics. Vol. 7, No. 1, 2019, pp. 23-26. doi: 10.11648/j.ejb.20190701.14

Received: May 22, 2019; Accepted: July 30, 2019; Published: August 20, 2019

\begin{abstract}
Fluorescence, absorption, and excitation spectroscopy have been widely utilised as probes to collect basic information about physical, chemical, and biological processes. In this study nitrogen laser $\left(\mathrm{N}_{2}\right)$ was used to induce emission in human teeth to distinguish between dental caries and sound teeth. Three samples of dental caries and one sample of sound teeth have been used to obtain fluorescence spectra illuminated with wavelengths of $337.1 \mathrm{~nm}$, pulse energy $0.04 \mathrm{~mJ}$ and pulse time $100 \mathrm{nsec}$. The absorbance of dental caries and sound teeth was determined using UV-Vis spectrophotometer. The result showed an emission of broadband in the visible region from $363 \mathrm{~nm}$ to $627 \mathrm{~nm}$; it provided an amount of information related to intrinsic fluorophores and allowed an accurate diagnosis by the use of the fluorescence intensity changes, it was observed significant decrease of the fluorescence signal intensity related to the carious stage in dental caries, while it was higher in the sound tooth spectrum indicates that the intensity is depending on the amount of decay. A definite diagnosis could be established based on the fluorescence intensity ratio. The future for laser induced fluorescence in diagnostic dentistry has been indicated as accurate and potentially applicable in a wide range in restorative dentistry, periodontology and endodontics.
\end{abstract}

Keywords: Dental Caries, Diagnostic, Fluorescence, Laser-matter Interactions, Laser Induced Fluorescence

\section{Introduction}

Laser-induced fluorescence (LIF) is the optical emission from molecules that have been excited to higher energy levels by absorption of photons. One of the most frequently used applications of LIF is measuring a biological preparation with a dye. A fluorescing dye is chosen which binds to specific structures inside a cell so that when the preparation is lit by a laser, an image of the structures can be made. Sometimes not even a dye is needed but already part of an organism, for example, photosynthesis [1].

Dental caries is a disease in which enamel and dentin are gradually eroded, due to the elimination of minerals by acid excreted from bacteria in tooth surface plaque. Dental caries is a chronic disease that occurs frequently.

Dental caries is a disease, which leads to the destruction of the tooth structure and eventually to infection of the dental pulp and even surrounding tissues. Factors contributing to the progression of the disease include diet (mainly fermentable carbohydrates), microbes, and the host (amount and constituents of the saliva, habits). The progression of dental caries lesions needs time. Fluoride protects the teeth from dental caries by influencing the tooth structure [2].

Dental caries is usually performed using a tool called explorer that catches as it moves across a tooth with a cavity. This method of detection has many limitations, including the instrumentation.

Laura E. Tam, DDS, et al in (2001), reviewed current knowledge concerning conventional and new diagnostic methods for occlusal caries. These methods have several limitations, particularly in their ability to diagnose early carious lesions. Part II examines new and emerging technologies that are being developed for the diagnosis of 
occlusal decay. Electrical conductance measurements and quantitative laser- or light-induced fluorescence represent significant improvements over conventional diagnostic methods, especially for in vitro applications and particularly with regard to sensitivity and reproducibility [3].

Ana Maria COSTA, Lillian Marley de PAULA, Ana Cristina Barreto BEZER in (2007), Evaluated the use of a laser autofluorescence device for detection of occlusal caries in permanent, was found that the laser detection method produced high values of sensitivity $(0.93)$ and specificity (0.75) and a moderate positive predictive value $(0.63)$. The laser device showed the lowest value of likelihood ratio (3.68). Kappa coefficient showed good repeatability for all methods. Although the laser device had an acceptable performance, this equipment should be used as an adjunct method to visual inspection to avoid false positive results [4].

Anttonen, Vuokko in (2007), used Laser autofluorescence in detecting and monitoring the progression of occlusal dental caries lesions and for screening persons with unfavourable dietary habits This study focused on the clinical use of laser autofluorescence compared to visual inspection (VI) for detecting and monitoring the progress of caries lesions during a one-year follow-up period and for screening subjects with unfavorable dietary habits causing demineralization of teeth [5].

Roxana Rangalet al in (2007), has attempted to emphasize the efficiency of laser- autofluorescence (AF) and fibre optic trans-illumination (FOTI) as complementary methods for diagnosing the early caries lesion. It has also demonstrated the interactive and didactic role of the Diagno dent pen [6].

Bennett T. Amaechia in (2009) described the various technologies available to aid the dental practitioners in detecting dental caries at the earliest stage of its formation, assessing the activities of the detected carious lesion, and quantitatively or qualitatively monitoring of the lesion over time. The need and the importance of these technologies were also discussed. The data discussed are primarily based on published scientific studies and reviews from case reports, clinical trials, and in vitro and in vivo studies [7].

Zangooei Booshehry et al, in (2010), evaluated different caries diagnostic methods, Clinical Visual Inspection, Fiber Optic Transillumination, Caries Indicter Dyes, and Fluorescent Methods [8].

Fardad Shakibaie, Roy George, L. J. Walsh, in (2011), proposed the principle of the fluorescent phenomenon, then explore the scientific background of fluorescent studies on the dental tissue. The laser-induced fluorescence can be used to detect and diagnose dental caries, calculus and bacterial biofilms in dental applications. Pini et al. used laser fluorescence to detect residual pulp tissue within the root canal, using a $308 \mathrm{~nm}$ wavelength ultraviolet laser, while Sarkissian and Le used 366, 405, and $440 \mathrm{~nm}$ wavelengths to distinguish remaining pulp tissue and bacteria from normal hard tissue in root canals. Most work using autofluorescence in dentistry has employed visible light as the excitation source [9].

Yao-Sheng Hsieh 1, 2, and et al in (2013) described the applications of dental optical coherence tomography (OCT) in oral tissue images, caries, periodontal disease and oral cancer. The background of OCT, including basic theory, system setup, light sources, spatial resolution and system provided. The comparisons between OCT and other clinical oral diagnostic methods are also discussed [10].

In this work laser-induced emission was used to distinguish between emission from dental caries and sound tooth and to investigate the relation between emission intensity and the amount of decay.

\section{Experimental Part}

\subsection{Samples}

Dental caries and sound tooth were studied in this work. Immediately after extraction, the teeth were fixed in formalin and rinsed out in distilled water, and sampled independently by specialist-dentist. The conditions of the dental caries were in different tooth disorders, namely, superficial cavity, medium depth cavity and deep cavitation, the control was the sound tooth; shown in Figures 1 and 2.

No formalin spectrum signals from the samples were recorded. The samples were kept in a proper environment. Each tooth from the samples was investigated by laserinduced fluorescence spectroscopy.



Figure 1. Dental Caries.

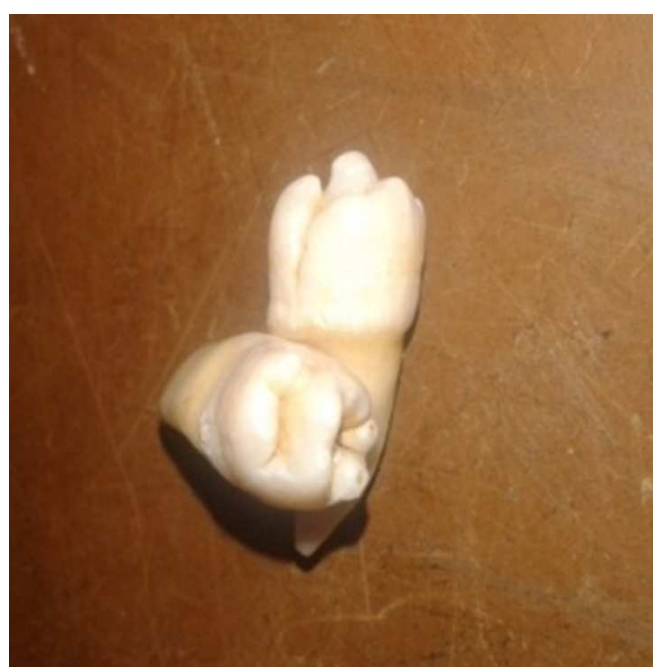

Figure 2. Sound Teeth. 


\subsection{Detection System}

The spectra were detected with a USB 2000+UV-VIS (PC2000, "Ocean Optics", Inc., Dunedin, FL, USA). A computer was utilised to control the system and to store and show data. The spectra were stored by the spectrometer specialized program (OOI Base, "Ocean Optics", Inc. Dunedin, FL, USA) and were analyzed and graphically represented with another computer program (Origin 5.0, Microcal Software, Inc., Northampton, MA, USA) shwon in Figure 3.

\subsection{Photoluminescence (PL) System}

Nitrogen laser was used as an excitation sources to obtain the fluorescence spectra $(337.1 \mathrm{~nm}, 14 \mathrm{~J}, 10-\mathrm{Hz}$ repetition rate). Four fluorescence spectra were detected, three from the dental caries from an intact area of the tooth and one from the sound tooth. The spectrum of the sound tooth surface was used as a reference of the spectral changes in the dental caries. Schematically the Photoluminescence experimental setup is shown in Figure 3.

Photoluminescence (PL) properties were determined using nitrogen $\left(\mathrm{N}_{2}\right)$ laser with a wavelength of $337.1 \mathrm{~nm}$, power $0.04 \mathrm{~mW}$, and repetition rate $100 \mathrm{nsec}$, shown in Figure 3.

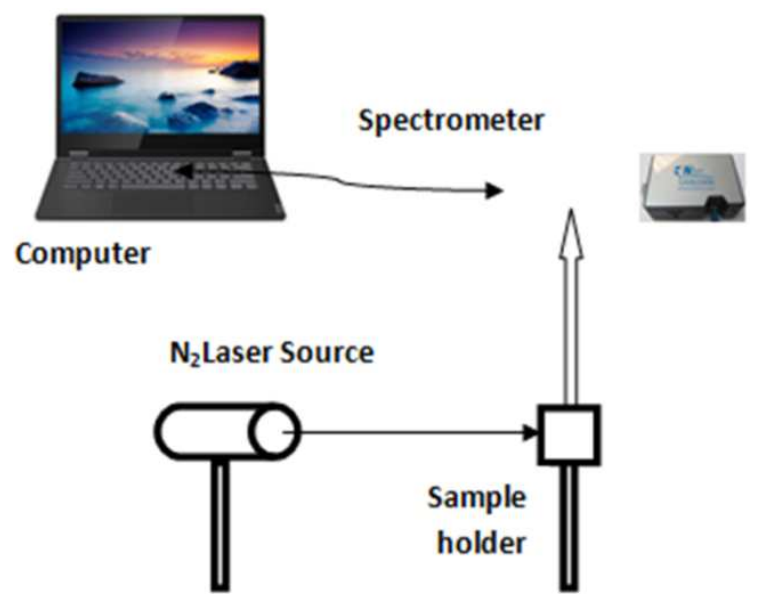

Figure 3. PL system.

\section{Results and Discussion}

The interaction between ultraviolet laser photons and the teeth was induced visible spectrum recorded by a USB spectrophotometer. Figure 4 shows the fluorescence spectra for the dental caries affected by different stages of caries, namely, superficial cavity, medium-depth cavity and deep cavitation, using nitrogen laser as an excitation source. Normalization was done to compare the spectral shape changes in Figure 5, the spectra were normalized with respect to the maximum intensity peak. The sound tooth spectrum consisted of a broadband (485-495 nm maximum) of fluorescence with one secondary maximum at $440 \mathrm{~nm}$; that of the carious tooth exhibited the same maxima at 490 and $440 \mathrm{~nm}$. This intensity peak decreased further for all carious areas depending on the stage, with deep cavitation displaying the weakest fluorescence. The secondary peak of the carious areas at $440 \mathrm{~nm}$ reduced for all carious areas depending on the carious stage too. No direct relationship was observed between the changes of the fluorescence spectra shape and the caries condition.

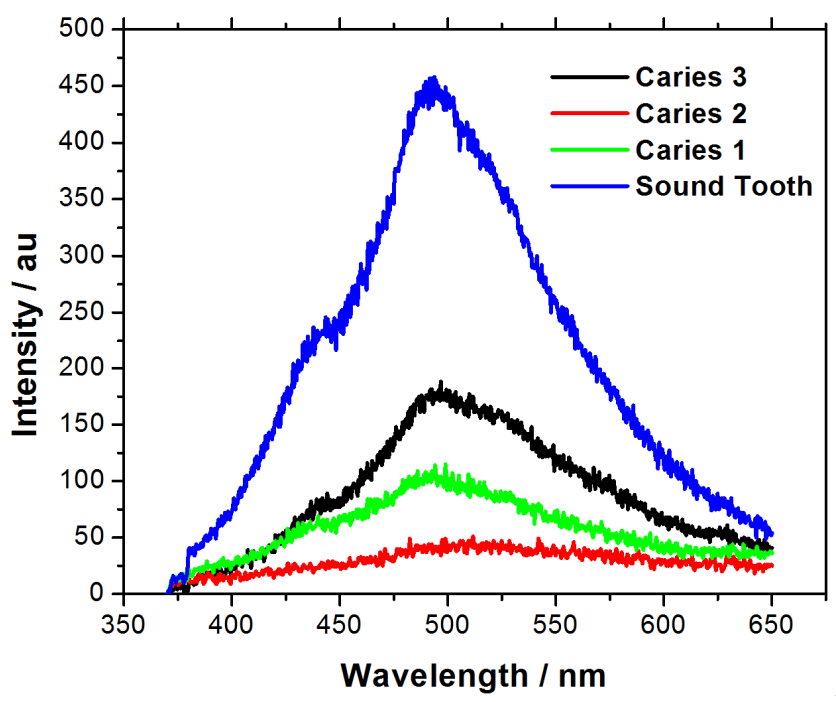

Figure 4. Spectra of dental caries and sound tooth (Excitation at $337.1 \mathrm{~nm}$ ).

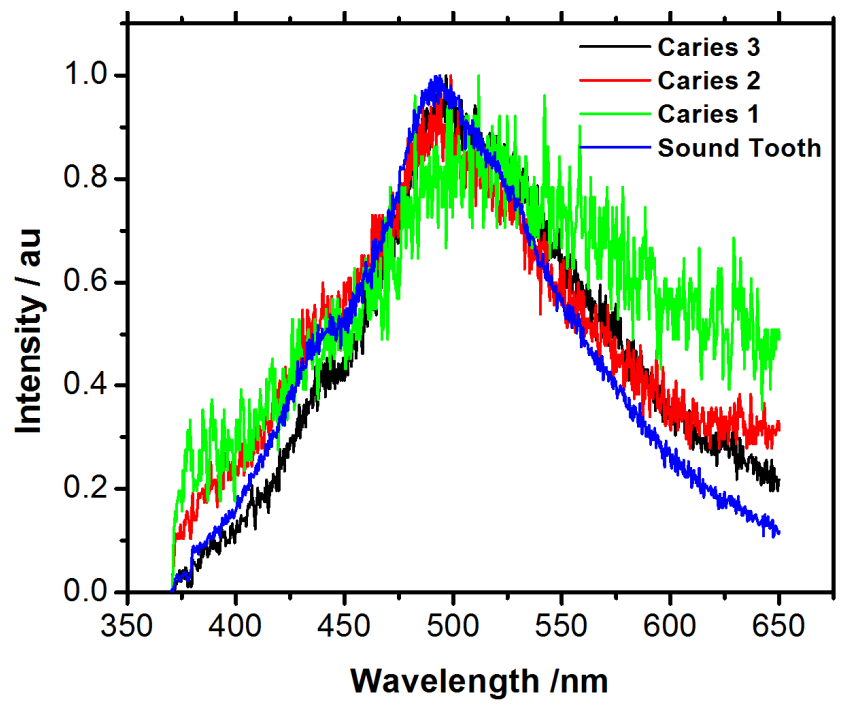

Figure 5. Comparison between spectra of a sound tooth and of different carious stages.

Figure 6 showed the emission spectra of the dental caries and sound tooth (sample 4) excitation at $337.1 \mathrm{~nm}$ treated and smoothed by origin program. The intensity of the broad peak at $(500) \mathrm{nm}$ of the caries 3 is $312.4 \mathrm{au}$, caries 1 is $230.5 \mathrm{au}$, and the intensity of the caries 2 is $166 \mathrm{au}$. These spectra show clearly that the intensity in the sound tooth (sample 4) is very high (364.42) au while in dental caries 3 the intensity is low (144.62) au in caries1the intensity is lower (69.6) au than caries 3 and in caries 2 the intensity is the lowest (52.47) au. Small shift was observed in the maximum emission position of the sample but it didn't carious stages.

The caries proportional inverse to intensity of induced emission. 
Table 1. Comparative of the dental caries and sound tooth.

\begin{tabular}{lllll}
\hline No. of sample & $\lambda_{\max }(\mathbf{n m})$ & Area & Fw. HM & I (a. u.) \\
\hline Sample1 & 502.9 & 8460.86 & 114.24 & 69.6 \\
Sample2 & 531.79 & 15295.38 & 273.81 & 52.47 \\
Sample3 & 513.28 & 11917.71 & 124.19 & 144.62 \\
Sample4 & 501.78 & 44938.24 & 115.58 & 364.42 \\
\hline
\end{tabular}

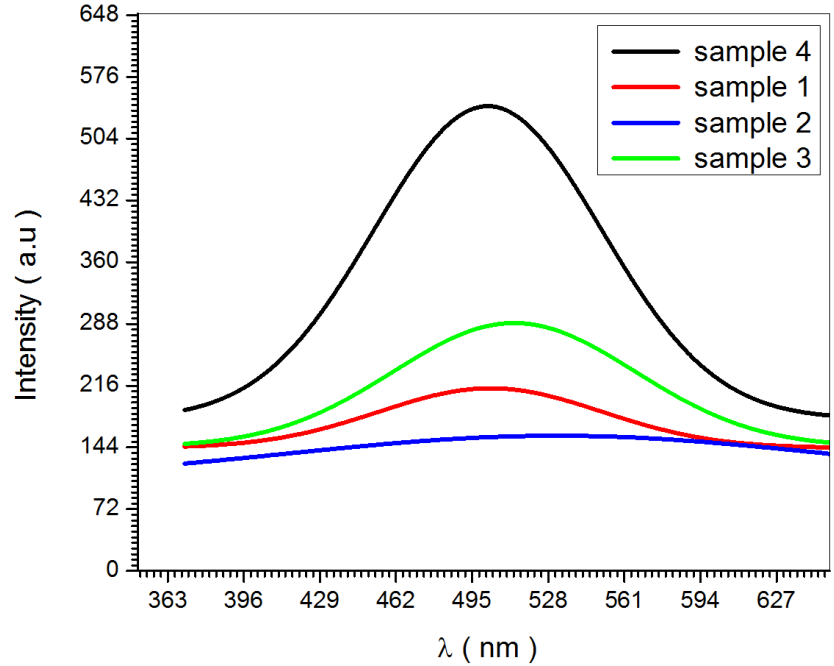

Figure 6. The emission spectra of the dental caries and sound tooth (Excitation at $337.1 \mathrm{~nm}$ ).

The results show that there is a different in the intensities of the emission peaks from the Luminescence of dental caries and sound tooth.

In this experimental study, it was observed significant differences between the fluorescence spectra of sound tooth and those of different carious stages. These differences can be attributed to differences in fluorophore content, but absorption and scattering of excitation and fluorescent light by the carious substance must also be taken into account.

The ability of laser-induced fluorescence spectroscopy was assessed to distinguish between initial caries and sound tooth and to classify the different stages of caries. The present study demonstrated the potential of the LIF technique to distinguish sound tooth from initial caries.

\section{Conclusions}

PE (photoemission) spectra of dental caries and the sound tooth have been obtained and investigated. All samples produce abroad band in the visible region.

The obtained results show different PE spectra from all samples. Comparing the PL spectra from the sound tooth with dental caries, it was found that the intensity is decreased in the caries tooth which indicates that the intensity is depending on the amount of decay.

\section{Acknowledgements}

Before of all, author renders my praise to God who offered me the health to perform this work. Authors would like to thank all members of the department of physics and laser institute at Sudan University of Science and Technology (SUST). Authors acknowledge the immense help received from the scholars whose articles are cited and included in references of this manuscript. The authors are also grateful to authors/editors/publishers of all those articles, journals and books from where the literature for this article has been reviewed and discussed.

\section{References}

[1] Kaminski, C., 2005. Fluorescence imaging of reactive processes. Zeitschrift für Physikalische Chemie, 219 (6), pp. 747-774.

[2] Hernández-Monjaraz, Beatriz, Edelmiro Santiago-Osorio, Edgar Ledesma-Martínez, Andrés Alcauter-Zavala, and Víctor Manuel Mendoza-Núñez. "Retrieval of a periodontally compromised tooth by allogeneic grafting of mesenchymal stem cells from dental pulp: A case report." Journal of International Medical Research (2018): 0300060518773244.

[3] Tam, L. E. and McComb, D., 2001. Diagnosis of occlusal caries: Part II. Recent diagnostic technologies. Journal of the Canadian Dental Association, 67 (8), pp. 459-464.

[4] Costa, A. M., Paula, L. M. D. and Bezerra, A. C. B., 2008. Use of diagnodentâ for diagnosis of non-cavitated occlusal dentin caries. Journal of Applied Oral Science, 16 (1), pp. 1823.

[5] Anttonen, Y., 2007. Laser fluorescence in detecting and monitoring the progression of occlusal dental caries lesions and for screening persons with unfavourable dietary habits. Oulun yliopisto.

[6] Yang, J. and Dutra, V., 2005. Utility of radiology, laser fluorescence, and transillumination. Dental clinics of North America, 49 (4), p. 739.

[7] Amaechi, B. T., 2009. Emerging technologies for diagnosis of dental caries: The road so far. Journal of applied physics, 105 (10), p. 102047.

[8] Booshehry, M. Z., Fasihinia, H., Khalesi, M. and Gholami, L., 2011. Dental Caries Diagnostic Methods. DJH, 2 (1).

[9] Shakibaie, F., George, R. and Walsh, L. J., 2011. Applications of laser induced fluorescence in dentistry. International Journal of Dental Clinics, 3 (3).

[10] Hsieh, Y. S., Ho, Y. C., Lee, S. Y., Chuang, C. C., Tsai, J. C., Lin, K. F. and Sun, C. W., 2013. Dental optical coherence tomography. Sensors, 13 (7), pp. 8928-8949. 